\title{
Influence of the aging model of lithium-ion batteries on the management of PV self-consumption systems
}

\author{
Alberto Berrueta, Julio Pascual, Idoia San Martín, Pablo Sanchis, Alfredo Ursúa \\ Department of Electrical and Electronic Engineering \\ Institute of Smart Cities \\ Public University of Navarre \\ Pamplona, Spain \\ alberto.berruetaunavarra.es
}

\begin{abstract}
Lithium-ion batteries are gaining importance for a variety of applications due to their improving characteristics and decreasing price. An accurate knowledge of their aging is required for a successful use of these ESSs. The vast number of models that has been proposed to predict these phenomena raise doubts about the suitability of a model for a particular battery application. The performance of three models published for a Sanyo 18650 cylindrical cell in a self-consumption system are compared in this work. Measured photovoltaic production and home consumption with a sampling frequency of 15 minutes are used for this comparison. The different aging predictions calculated by these three models are analyzed, compared and discussed. These comparison is particularized for two management strategies. The first of them maximizes the self-consumption $P V$ energy, while the second reduces the maximum power peak demanded from the grid.
\end{abstract}

Keywords-Lithium-ion battery; aging model; renewable energy; microgrid; battery management

\section{INTRODUCTION}

Due to both environmental and economical issues, the use of renewable energy increases around the World. Significant efforts are dedicated to the increasing of renewable energy share in the electricity grid. The role of energy management in these grids is gaining attention, leading to new concepts, such as microgrids or smart grids. Energy storage systems (ESS) are essential elements to allow for the energy management in an electricity grid. Among the available ESS, lithium-ion batteries are an attractive option, given their outstanding power capability, high energy density and decreasing price [1].

One of the most critical aspects that need to be accounted for a successful application of Li-ion batteries is the battery aging. Actually, the aging processes determine the battery lifetime, which is directly related to the profitability of the system, and affect battery capacity and resistance, the main variables of a battery electrical model [2]. The main chemical reactions that contribute to battery aging depend on the chemical composition of the battery electrodes and electrolyte, the manufacturing process and the operating conditions. The full understanding of the aging phenomena is still under

We would like to acknowledge the support of the Spanish State Research Agency (AEI) and FEDER-UE under grants DPI2013-42853-R, DPI201680641-R and DPI2016-80642-R; of Government of Navarra through research project PI038 INTEGRA-RENOVABLES; and the FPU Program of the Spanish Ministry of Education, Culture and Sport (FPU13/00542). research, and a number of chemical studies and aging models are published every month in the top rated research journals. Therefore, various parameters are proposed to determine the lifetime of the battery. The selection of an aging model, which is a tool to design the battery management strategy, should depend on the particular battery under study. For instance, if the model predicts deep charge-discharge cycles to produce high battery degradation, the management algorithm should avoid this kind of cycles.

The aim of this contribution is to analyze the diversity of simulation results obtained with the application of different $\mathrm{Li}$ ion battery aging models, as well as the implications of this diversity in the design of the battery management strategy. With this purpose, three published models for the same Li-ion battery are identified, as summarized in Section II. This battery aging models are applied in Section III to a typical home photovoltaic (PV) system, in which the battery is managed based on two different strategies. The first one aims at a maximization of the PV self-consumption, while the second one reduces the maximum power peak demanded from the electricity grid. In Section IV, the different results obtained by means of each battery model using both strategies are analyzed and compared. Finally, the conclusions of this study are stated in Section V.

\section{LI-ION BATTERY AGING MODELS}

The battery analyzed in this contribution is build by the series and parallel connection of the Sanyo cell UR18650E which is a cylindrical cell, with a rated capacity of $2.05 \mathrm{Ah}$. Its cathode is made of $\mathrm{Li}(\mathrm{NiMnCo}) \mathrm{O}_{2}$ (NMC), while the anode material is graphite. The three battery aging models studied in this contribution are explained in the following subsections.

\section{A. Model I: based on equivalent full cycles}

One of the most straightforward and common ways of modeling the battery aging is based on the number of equivalent charge-discharge full cycles (EFC), defined as follows:

$$
E F C=\int \frac{|i|}{2 \cdot C} \cdot d t
$$

being $i$ the battery current, $C$ its capacity and $t$ time.

Battery datasheets usually provide this kind of aging models, given their ease of use. Fig. 1 is reproduced from the 


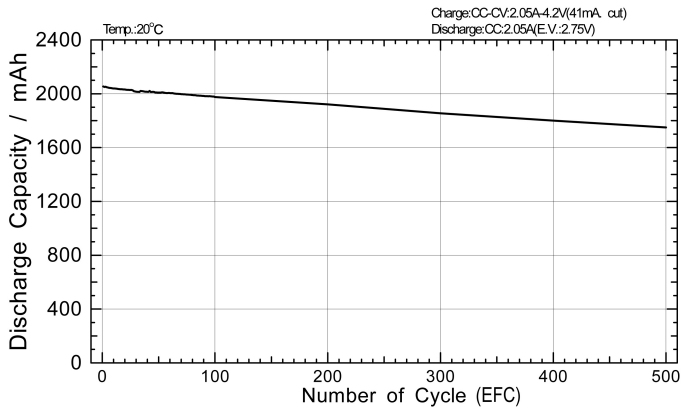

Fig. 1. Evolution of the battery capacity as a function of EFC, reproduced from the datasheet provided by the manufacturer [3].

datasheet of the studied battery [3], and shows the relationship between the battery capacity and the number of equivalent full cycles. As indicated at the top of the figure, these data are valid for a temperature of $20^{\circ} \mathrm{C}$.

The charge-discharge cycles are a main variable for the determination of the aging phenomena, given that the changes in the electrode crystal structure required for the charging and discharging of the battery induce irreversible parasitic reactions that degrade the battery. However, the main drawbacks of this kind of models are that they are valid for a single temperature level and that variables such as depth of discharge (DOD), battery current or battery voltage, which also influence the degradation processes, are not taken into account.

\section{B. Model II: accounting for voltage, depth of discharge and} time

Most of Li-ion battery aging models divide the aging phenomena into two independent terms, named calendar and cycle aging. Calendar aging does not depend on battery use, while cycle aging represents only phenomena that are triggered by charge-discharge cycles. Both effects are summed up in order to calculate the overall battery aging.

An aging model based on these two terms for the Sanyo UR18650E cell was published by Sauer and co-workers [4]. The authors analyze both capacity fade and impedance rise by means of the following per-unit expressions:

$$
\begin{aligned}
& C=1-\alpha_{\text {cap }} \cdot t^{0.75}-\beta_{\text {cap }} \cdot \sqrt{E F C} \\
& R=1+\alpha_{\text {res }} \cdot t^{0.75}+\beta_{\text {res }} \cdot E F C
\end{aligned}
$$

where $t$ is expressed in days and $\alpha_{i}$ and $\beta_{i}$ (being $i=c a p$ or res) are the variables used to model calendar and cycle aging phenomena respectively. $\alpha_{i}$ are functions of the battery voltage and temperature, while $\beta_{i}$ are functions of DOD and average cycle voltage.

\section{Model III: accounting for current, SOC and time}

Wang et al. [5] also present an aging analysis of a Sanyo UR18650 cell based on a wide set of experiments. In contrast with the previously-explained MODEL II, capacity fade and impedance rise are grouped together in the variable called

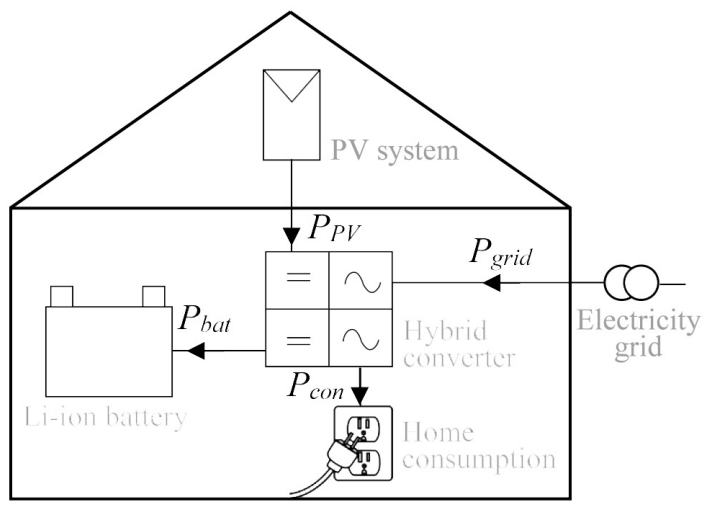

Fig. 2. Schematic diagram of the self-consumption system.

$S O H$ (state of health) and the following expression is proposed:

$$
\begin{aligned}
\Delta S O H= & -\left[0.0008 \cdot \exp \left(0.3903 \cdot\left|\frac{i}{C}\right|\right)\right] \cdot 2 \cdot C \cdot E F C \\
& -14.876 \cdot t^{0.5} \cdot \exp \left(\frac{-24500}{R \cdot T}\right)
\end{aligned}
$$

In this expression, the term $\frac{i}{C}$ is the normalized battery current, $2 \cdot C \cdot E F C$ is the ampere-hour throughput, $t$ is expressed in days, $T$ in $\mathrm{K}$ and $R$ is the gas constant.

\section{GRID-CONNECTED PV SELF-CONSUMPTION USING BATTERIES}

The models explained in Section II are applied in this work to predict the aging of a battery used as ESS of an experimental self-consumption PV installation, whose schematic is shown in Fig. 2. This is a grid-connected rooftop PV system with a peak power of $1.75 \mathrm{~kW}$ used to feed the electricity demand of a five-member family home located in a village close to Pamplona, in the north of Spain. This house is connected to the electricity grid, which is used to feed its energy demand during the periods when no PV electricity is available. A $6.5 \mathrm{kWh}$ battery is used to increase the PV self consumption, thereby reducing the energy demanded from the electricity grid. This battery size has been chosen as a realistic capacity of commercial home batteries, such as the LG Chem Resu6.5. The constraint of no power injected into the grid is imposed to this installation, given that it is a typical requirement in a number of European grid codes $\left(P_{\text {grid }} \geq 0\right.$ in Fig. 2). As represented in Fig. 2, the power demanded by the home $\left(P_{c o n}\right)$ comes either from the direct PV generation $\left(P_{P V}\right)$, from the discharging battery $\left(-P_{b a t}\right)$, or from the electricity grid $\left(P_{\text {grid }}\right)$ when there is no other option. Therefore, the power balance can be written as follows:

$$
P_{\text {con }}=P_{P V}-P_{b a t}+P_{\text {grid }}
$$

The profitability, performance and aging of the battery depends on the strategy used to manage the ESS. A domestic ESS can be managed according to different strategies. For 
example, it can be used to increase the PV self-consumption, to reduce the maximum power peak absorbed from the grid, to soften the grid power profile, to provide grid services etc. Two of these strategies are proposed in this contribution in order to contrast the battery aging predicted by each model. PV generation and home consumption power measurements during a complete year are utilized for this study, and the following two strategies are programmed for the management of the battery.

The first strategy, shown in Fig. 3 (a), aims at maximizing the PV self-consumption. With this purpose, the battery is simulated to be charged while there is a surplus PV generation (pink areas in Fig. 3 (a)) and discharged when there is not enough generation to feed the home consumption (blue areas in the figure). Electricity is demanded from the grid only when the battery is fully discharged and the home requires more power, as shown by the gray area in Fig. 3 (a). Only $25 \%$ of the total energy demanded by the home during the studied year needs to be taken from the grid when using this management strategy. However, the maximum power peak that the grid needs to provide is as high as $4 \mathrm{~kW}$. Given that the electricity network is sized to be able to manage the highest power peak, a fixed price per available $\mathrm{kW}$ is applied in most electricity tariffs. This is the reason why ESSs are sometimes used to reduce this maximum demanded power, thereby reducing the electricity bill, as proposed in the second strategy analyzed in this contribution.

The aim of the second strategy is to reduce the maximum power peak demanded from the grid, even though it entails a slight reduction in the PV self-consumption. With this purpose, the battery is charged when there is extra PV production and discharged when required, as explained for the first strategy. The difference is that a limiting SOC is set at $25 \%$, and the battery discharging is stopped at this point. If more electricity is demanded by the home, it is taken from the electricity grid. Only if the demanded power is higher than $1 \mathrm{~kW}$, the extra power is taken from the battery. When the battery is discharged below $25 \%, 1 \mathrm{~kW}$ is constantly consumed from the grid in order to recover the $25 \%$ SOC of the battery, as shown in Fig. 3 (b). With this management strategy, the maximum peak demanded from the grid is decreased to a third of the value achieved by the first strategy $(1.3 \mathrm{~kW})$ at the expense of a reduction in the PV self-consumption from the $75 \%$ achieved with the first strategy to $72 \%$ obtained with the second one.

\section{MODEL COMPARISON}

The aging of the Li-ion battery in such a PV selfconsumption application predicted by the three aging models mentioned in Section II are herein contrasted. For the sake of comparability, given that MODEL I is particularized for a temperature of $20{ }^{\circ} \mathrm{C}$, this temperature is assumed for the other two models. The number of equivalent full cycles (EFC) demanded from the battery during the 365 days of the year calculated by means of Equation 1 is $E F C=210$. $E F C$ is the only variable required for MODEL I. However, the DOD, and average SOC, current and voltage of each cycle are needed
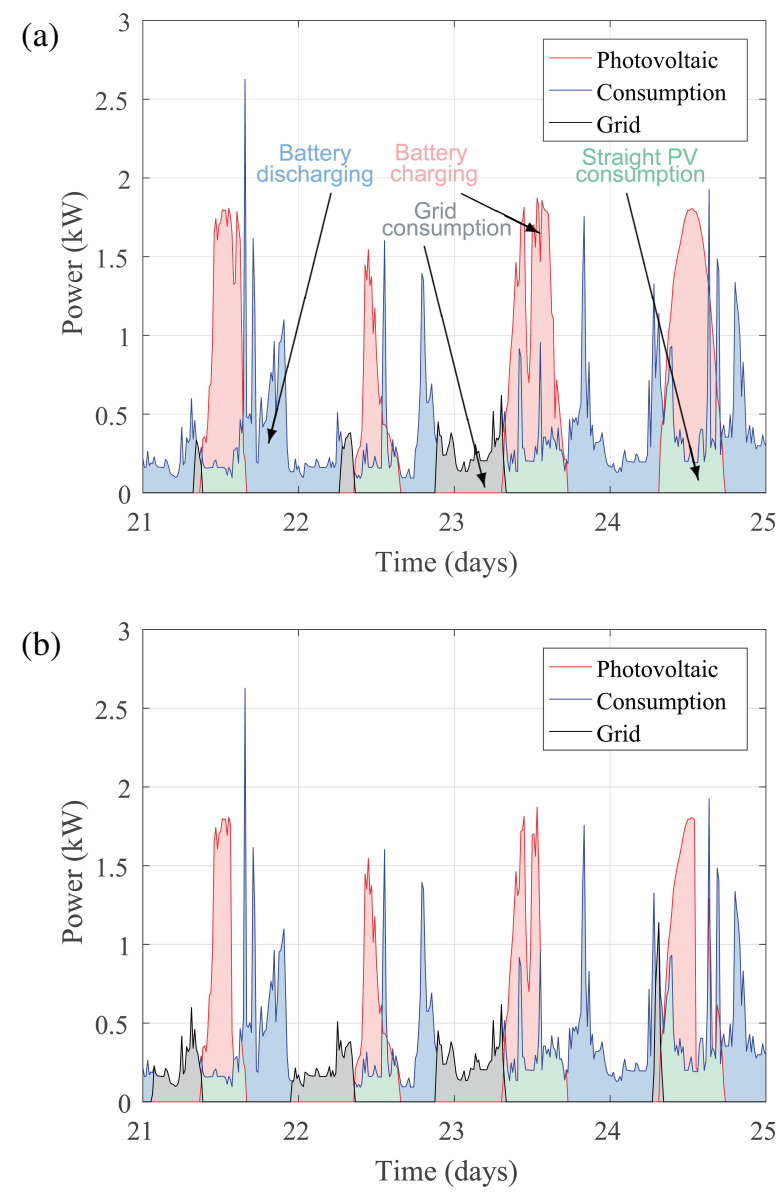

Fig. 3. Main power flows of the PV self-consumption system during four days in January: photovoltaic generation, home consumption and power demanded from the electricity grid using different objectives for the management strategy: (a) maximum PV self-consumption and (b) minimum power peak demanded from the electricity grid.

for Model II. Meanwhile, ModEL III requires the equivalent full cycles, current, temperature and time as input variables. The separation of the superimposed battery cycles resulting from the analyzed application, as required by MODEL II, is tackled by means of the rain-flow algorithm [6]. This algorithm separates the evolution of the battery SOC into equivalent full cycles with different depth of discharge (DOD). Fig. 4 shows the DOD distribution of the battery charge-discharge cycles. The sum of all these EFCs is 210, as stated above.

Fig. 5 represents the state of health $(\mathrm{SOH})$ predicted by each of the aging models along the year using both management strategies: the maximization of the PV self-consumption (a) and the minimization of the peak power demanded from the grid (b). It is noteworthy that the end of life of a Li-ion battery is considered to be around $S O H=0.8$. This consideration is due to the combination of two effects: on the one hand, the battery impedance rise reduces the maximum manageable power and, on the other hand, the capacity fade and impedance rise are accelerated for $S O H<0.8$, resulting in a faster battery degradation.

The aging characteristics predicted by MODEL I are the 


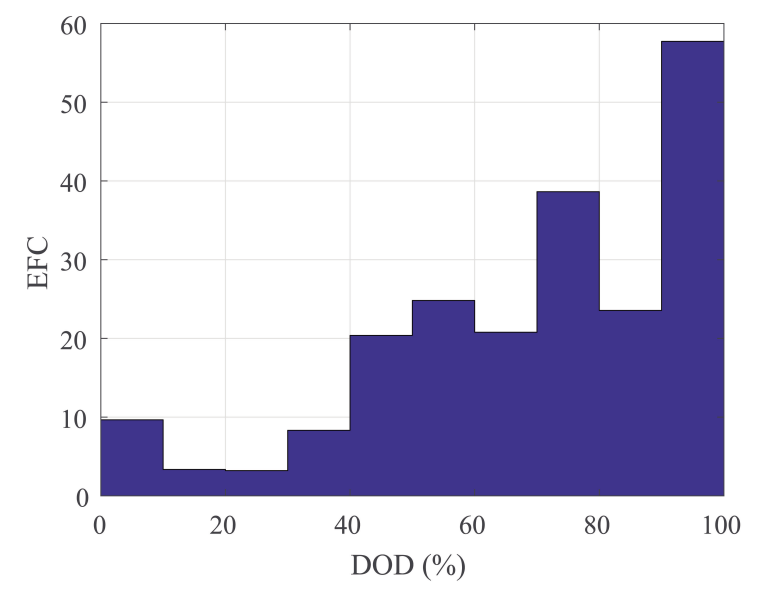

Fig. 4. Histogram of the equivalent full cycles (EFC) classified based on their depth of discharge (DOD) for the maximization of the PV self-consumption.

slowest of the three studied predictions regardless of the management strategy. The reason for this optimistic forecast is that the cycles considered by this model are standard cycles, and important variables, such as DOD, current or voltage are disregarded. Therefore, even though MODEL I is the aging model provided by the battery manufacturer, care should be taken when using a battery for a non-standard application, such as the one described in this work. These uneven results highlight the importance of a Li-ion battery model for an accurate prediction of its lifetime.

The predictions provided by MoDEL II and MoDEL III are also different, due to the distinct variables considered by each of them: while MODEL II predicts the battery aging based on battery voltage, DOD and time, MODEL III uses battery current, average SOC and time as input variables. The aging prediction provided by MODEL II is the most pessimistic when the PV self-consumption is maximized (Fig. 5 (a)). The battery $\mathrm{SOH}$ predicted by this model at the end of the year is $\mathrm{SOH}=$ 0.83 . This is due to the fact that a large number of EFC have a DOD $>90 \%$, as shown in Fig. 4, which is an accelerating aging factor based on MODEL II. Actually, this model predicts a battery $\mathrm{SOH}$ of 0.85 at the end of the year for the strategy that minimizes the peak power demanded from the grid. The reason for this increased $\mathrm{SOH}$ is that the battery is prevented for discharges below $\mathrm{SOC}=25 \%$, which contributes to a longer lifetime.

Finally, the SOH predicted by MODEL III is close to the prediction provided by MODEL II, and suffers no significant variation when the management strategy is changed. This lack of variation is due to the fact that DOD is not considered as an accelerating aging parameter by MODEL III. Therefore, if an accurate $\mathrm{SOH}$ prediction is required, a close analysis of the battery characteristics is needed in order to determine the main parameters that drive its aging processes.

Note that this battery was manufactured around year 2012 and it was not designed for stationary, self-consumption applications. This is the reason for the fast aging processes that degrade the battery in a year of normal operation. Important
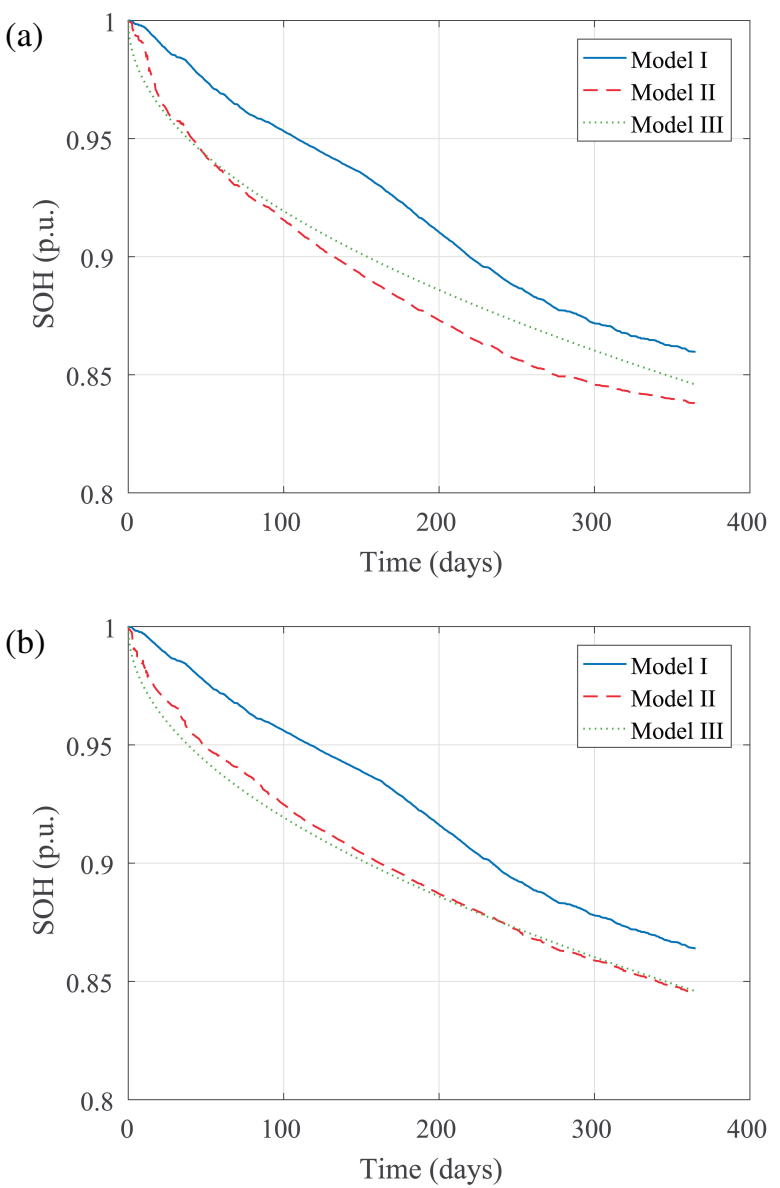

Fig. 5. SOH predicted by each of the studied models for a year of battery operation for the two analyzed management strategies: (a) maximum PV selfconsumption and (b) minimum power peak demanded from the electricity grid.

improvements in the field of Li-ion batteries have been carried out, and specific batteries for stationary applications have been manufactured, leading to a noticeable increase in the lifetime of these ESSs up to several thousands of cycles nowadays [5], [7], [8].

\section{CONClusions}

The aging phenomena have been identified as a main issue related to Li-ion batteries. Given the wide variety of Li-ion technologies and battery applications, a vast collection of aging models are published in research journals and battery datasheets. Three such models of a Sanyo UR18650 cell, based on different battery variables and with diverse levels of complexity, are herein capitulated and compared for a realistic, domestic PV self-consumption system. Moreover, the studied ESS has been managed by means of two algorithms aimed at two different objectives: the maximization of the PV self-consumption and the minimization of the peak power consumed from the grid. The predictions of the battery lifetime provided by these three models have substantial differences, leading to the conclusion that the selection of the battery aging model is an issue that needs to be carefully addressed. 
Moreover, among the three aging models, the one provided by the cell manufacturer in the product datasheet is the most optimistic one.

The prediction of battery aging provided by two of the three studied models for two different management strategies are similar, while the other model predicts slower degradation if the battery is used to reduce the maximum power peak consumed from the grid. Therefore, the selection of a suitable battery model needs to be taken into account for the design of a management strategy. If an accurate representation of the battery aging is achieved, the lifetime of the ESS can be enlarged, thereby improving the profitability of the system.

It is also noteworthy that operating temperature is a main parameter for battery aging. It has not been analyzed in this contribution given that the aging model provided by the manufacturer datasheet is particularized for $20{ }^{\circ} \mathrm{C}$. However, in a real study of battery aging, operating temperature needs to be carefully analyzed.

Therefore, the main conclusion reached by this contribution is that, previous to the design and selection of an aging model, an analysis of the battery application should be made and the model should be selected or designed to have the highest accuracy for the studied application. The notorious difference of battery SOH predicted by different aging models can significantly deviate the calculation of the durability and profitability of the studied Li-ion battery.

\section{REFERENCES}

[1] P. Cazzola and M. Gorner, "Global EV outlook 2016," International Energy Agency, Technical report, 2016.

[2] A. Berrueta, A. Urtasun, A. Ursúa, and P. Sanchis, "A comprehensive model for lithium-ion batteries: From the physical principles to an electrical model," Energy, vol. 144, pp. 286-300, 2018.

[3] Cell Type UR18650E. Specifications, Sanyo Electric Co., Ltd. [Online]. Available: https://www.master-instruments.com.au/file/62415/ 1/Sanyo-UR18650E.pdf

[4] J. Schmalstieg, S. Käbitz, M. Ecker, and D. U. Sauer, "From accelerated aging tests to a lifetime prediction model: Analyzing lithium-ion batteries," in 2013 World Electric Vehicle Symposium and Exhibition (EVS27), Nov 2013, pp. 1-12.

[5] J. Wang, J. Purewal, P. Liu, J. Hicks-Garner, S. Soukazian, E. Sherman, A. Sorenson, L. Vu, H. Tataria, and M. W. Verbrugge, "Degradation of lithium ion batteries employing graphite negatives and nickel-cobaltmanganese oxide + spinel manganese oxide positives: Part 1, aging mechanisms and life estimation," Journal of Power Sources, vol. 269, pp. $937-948,2014$.

[6] D. Jiang, "A sequence retainable iterative algorithm for rainflow cycle counting," SAE International Journal of Materials and Manufacturing, vol. 7, no. 1, pp. 108-114, 2014.

[7] J. Purewal, J. Wang, J. Graetz, S. Soukiazian, H. Tataria, and M. W. Verbrugge, "Degradation of lithium ion batteries employing graphite negatives and nickel-cobalt-manganese oxide + spinel manganese oxide positives: Part 2, chemical-mechanical degradation model," Journal of Power Sources, vol. 272, pp. 1154 - 1161, 2014.

[8] M. Petit, E. Prada, and V. Sauvant-Moynot, "Development of an empirical aging model for li-ion batteries and application to assess the impact of vehicle-to-grid strategies on battery lifetime," Applied Energy, vol. 172, pp. 398 - 407, 2016. [Online]. Available: http://www.sciencedirect.com/science/article/pii/S0306261916304500 